\title{
Antibiotic resistance patterns of group B streptococcal clinical isolates
}

\author{
Jose A. Simoes ${ }^{1,2}$, Alla A. Aroutcheva ${ }^{1}$, Ira Heimler ${ }^{1}$ and Sebastian Faro ${ }^{1,3}$ \\ ${ }^{1}$ Department of Obstetrics and Gynecology, Rush-Presbyterian-St. Luke's Medical Center, Chicago, IL \\ ${ }^{2}$ Department of Obstetrics and Gynecology, State University of Campinas, SP, Brazil \\ ${ }^{3}$ The Woman's Hospital of Texas, Houston, TX
}

Objectives: To determine the in vitro resistance of group B streptococcus (GBS) to 12 antibiotics. To determine if there has been any decrease in sensitivity to the penicillins or other antibiotics currently used for GBS chemoprophylaxis in pregnant women. Find suitable alternative antibiotics to penicillin. Find an antibiotic that will have minimal selective pressure for resistance among the endogenous resident vaginal microflora.

Methods: The antibiotic susceptibility profiles of 52 clinical isolates of GBS were evaluated to 12 antibiotics: ampicillin, azithromycin, cefamandole, cefazolin, ceftriaxone, ciprofloxacin, clindamycin, erythromycin, nitrofurantoin, ofloxacin, penicillin and vancomycin. Antibiotic sensitivities were determined using disk diffusion and microdilution methods according to the guidelines of the National Committee for Clinical Laboratory Standards (NCCLS).

Results: All isolates were sensitive to vancomycin, ofloxacin, ampicillin, ciprofloxacin, nitrofurantoin and penicillin. However, the following number of clinical isolates exhibited intermediate or decreased sensitivity, nine (I7\%) to ampicillin, eight (I5\%) to penicillin, I4 (32\%) to ciprofloxacin and one (2\%) to nitrofurantoin. Thirty-one percent of the isolates were resistant to azithromycin and ceftriaxone, $19 \%$ to clindamycin, $15 \%$ to cefazolin and $13 \%$ to cefamandole. Eighteen (35\%) of the clinical isolates tested were resistant to 6 of the 12 antibiotics tested.

Conclusions: The relatively high rates of resistance for 6 of the 12 antibiotics tested suggest that for women allergic to penicillin and colonized with GBS, antibiotic sensitivities to their isolates should be determined. The antibiotic selected for intrapartum chemoprophylaxis should be guided by the organism's antibiotic sensitivity pattern. Patients with GBS bacteriuria should be treated with nitrofurantoin.

Key words: Group B StrePtococcus; Resistance; ANTIBIOtics; Intrapartum Prophylaxis

\section{INTRODUCTION}

Group B streptococcus (GBS) continues to be an important cause of both infection and significant morbidity and mortality in newborns, and pregnant and non-pregnant women ${ }^{1-3}$. Vaginal colonization with GBS reportedly occurs in $5-35 \%$ of women ${ }^{4-7}$. Thus far GBS has not posed a significant problem for women undergoing gynecologic surgery; however, with the increase in gynecologic surgery occurring in the older population and the likelihood of these women having chronic illnesses, GBS poses a potentially significant threat for the development of postoperative infection morbidity and mortality.

Correspondence to: Sebastian Faro, Department of Obstetrics and Gynecology, The Woman's Hospital Texas, 7600 Fannin, Ste. 1160, Houston, TX 77054 
Although guidelines for preventing perinatal transmission of GBS to newborns have been published, early onset infection continues to be a problem. In the United States approximately 2200 early onset infections occur yearly ${ }^{8}$. The incidence of early onset GBS infection from 1998-1999 was 0.78/1000 live births in African American infants, 0.5/1000 among Hispanic infants and 0.3/1000 among Caucasian infants?.

In a Canadian study, the incidence of GBS in pregnant women was 41/100 000 and 4.1/100 000 in non-pregnant adults ${ }^{10}$. In this study $58.5 \%$ of the cases occurred in women and $14.2 \%$ were associated with pregnancy; $44.2 \%$ of the infections occurred in non-pregnant women. A similar study conducted in Atlanta, Georgia found an increasing trend in GBS-invasive disease; in 1982 there were 2.4 cases/100 000 population and in 1993 there were 5.9/100 $000^{2}$. In 1992 the incidence of GBS in pregnant women in Atlanta, Georgia was 22/100 000 and it rose to $66.4 / 100000$ in $1993^{2}$. The spectrum of serious disease caused by GBS in all age groups initiated the development of a vaccine $^{11-14}$.

This study was conducted because of the significant morbidity and mortality associated with GBS colonization and infection in newborn infants, as well as pregnant and non-pregnant women. Additionally, $13 \%$ of women are allergic to penicillin ${ }^{5,15,16}$. This should be considered along with the fact that vaginal colonization rates in pregnant and non-pregnant women are similar. This and the fact that overuse of antibiotics leads to the selection of resistant strains were the impetus for the study. Because of the high rate of antibiotic exposure for a variety of suspected bacterial infections, especially the $\beta$-lactam antibiotic, macrolides and quinolones, the antibiotics select for resistant strains among the numerous bacteria that make up the endogenous resident microflora of the vagina. Recent studies demonstrated that some GBS clinical isolates exhibited intermediate or decreased sensitivity, in vitro, to penicillins ${ }^{17-19}$. This decreased sensitivity should not be interpreted as resistance but instead be considered sensitive because it is not true resistance. However, this decrease in sensitivity to penicillin warrants monitoring of GBS for the possible development of resistance to penicillins. Thus, this study was performed to determine the prevalence of GBS strains among clinical isolates that have an intermediate sensitivity to the penicillins. Testing the response of clinical isolates to a variety of antibiotics would allow for alternative choices to be used for prophylaxis as well as treatment of GBS infection for individuals allergic to penicillin.

\section{METHODS}

A total of 52 GBS clinical isolates were examined from strains stored at $-70^{\circ} \mathrm{C}$ in skim milk (Difco Laboratories, Detroit) in the Obstetric and Gynecology Infectious Disease Research Laboratory collection. These isolates were collected from 1998 to 1999 . The clinical isolates were obtained from 45 non-pregnant women (35 vaginal, 10 rectal) and 7 newborns. Because all strains were stick isolates, each was subcultured three times on 5\% sheep blood agar plates (Remel Microbiology Products, Lenexa, KS). All isolates were confirmed as GBS on the basis of being Gram-positive cocci, catalase negative and having a positive latex agglutination assay (Patho Dx Strep Grouping, DPC, Los Angeles, CA) prior to initiating antibiotic susceptibility testing.

All procedures for disk susceptibility were performed in triplicate according to the methodologies described in the National Committee for Clinical Laboratory Standards (NCCLS) ${ }^{20}$. Fresh subcultures of GBS were used after overnight growth $(16 \mathrm{~h})$ on blood agar plate. The inoculum was prepared by suspending several of the colonies in sterile phosphate buffered saline $(\mathrm{pH}$ 7.2) to achieve a turbidity of 0.5 McFarland standard, determined by nephelometry. This resulted in a suspension containing approximately $1-2 \times 10^{8} \mathrm{CFU} / \mathrm{ml}$. A sterile cotton swab was dipped into the bacterial suspension, elevated above the liquid and rotated several times against the inside wall of the tube to remove excess inoculum. The swab was used to inoculate a $150 \mathrm{~mm}$ diameter Mueller-Hinton agar plate supplemented with 5\% defibrinated sheep blood. A second Mueller-Hinton agar plate without sheep blood was inoculated with a similar inoculum. The comparison of Mueller-Hinton 
with and without $5 \%$ sheep blood was performed to determine if the sheep blood had an essential effect on the antibiotic assay.

Eleven disks (Remel Microbiology Products, Lenexa, KS) were dispensed onto the surface of the inoculated agar plate. The antibiotics tested were: $10 \mu \mathrm{g}$ ampicillin; $15 \mu \mathrm{g}$ azithromycin; $30 \mu \mathrm{g}$ cefamandole; $30 \mu \mathrm{g}$ cefazolin; $30 \mu \mathrm{g}$ ceftriaxone; $5 \mu \mathrm{g}$ ciprofloxin; $2 \mu \mathrm{g}$ clindamycin; $15 \mu \mathrm{g}$ erythromycin; $5 \mu \mathrm{g}$ ofloxacin; $30 \mu \mathrm{g}$ vancomycin; and 10 units of penicillin G. One disk containing $300 \mu \mathrm{g}$ nitrofurantoin (Decton Dickinson Microbiology Systems, Cockeysville, MD) was placed onto the surface of a separate small agar plate. All plates were inoculated with GBS and incubated at $35^{\circ} \mathrm{C}$ under $5 \% \mathrm{CO}_{2}$ atmosphere for $20 \mathrm{~h}$ before they were read.

The zones of growth inhibition were measured to the nearest whole millimeter using a sliding caliper. The sizes of the inhibition zones were graded according to the NCCLS ${ }^{20}$. Each isolate was classified as susceptible, intermediate or resistant to each antibiotic tested. Minimum inhibitory concentrations (MIC) were determined by the broth microdilution method recommended by the NCCLS ${ }^{21}$. Cation-adjusted Mueller-Hinton broth (Remel Microbiology Products, Lenexa, KS) supplemented with 5\% lysed horse blood was used. Suspensions of GBS with a turbidity equivalent to a $0 / 5 \mathrm{McF}$ arland standard were prepared as previously described. These suspensions were further mixed with the supplemental broth at a 1:200 dilution to obtain a final inoculum concentration of $5-10 \times 10^{5} \mathrm{CFU} / \mathrm{ml}$. They were then dispersed into sterile microdilution test plates (Honeycomb microwell plate, Labsystems, Finland) prepared with different concentrations of the antibiotic to be assayed. The plates were incubated in ambient air at $35^{\circ} \mathrm{C}$ in a Bioscreen Analyzer System (Labsystems, Finland). The optical density of each microwell was measured automatically at $4-\mathrm{h}$ intervals for $24 \mathrm{~h}$. The MIC was based on the lowest concentration of each antibiotic showing complete inhibition of growth. The isolates were considered susceptible or resistant according to the 1999 interpretive standards of the NCCLS ${ }^{21}$. Two systems of antibiotic susceptibility, disk diffusion and microdilution or MIC, were used to determine if there was agreement in the interpretation of results between the two systems. This was especially true for those values interpreted as intermediate. In case of discordance between the results, the microdilution was considered the most reliable system.

Vaginal strains resistant and sensitive to clindamycin were screened for genotype. The DNA extraction and genotyping were determined by pulse-field gel electrophoresis. Rapid preparation method of bacterial DNA for pulse-field gel electrophoresis was used as a standard procedure with some modification suitable for $\mathrm{GBS}^{22}$. Isolates were grown overnight on $5 \%$ blood agar. All colonies grown on the blood agar were harvested and suspended in $2.5 \mathrm{ml}$ of brain-heart infusion broth (Remel Microbiology Products, Lenexa, KS) and centrifuged. The pellet of cells was resuspended in $0.5 \mathrm{ml}$ of $1.6 \%$ melted agarose and pipetted into a plug mold to solidify. Then agarose plugs were incubated sequentially in $3 \mathrm{ml}$ of $1 \mathrm{X}$ lysis solution ( $5 \mathrm{mg} / \mathrm{ml}$ lysozyme containing $30 \mu \mathrm{g} / \mathrm{ml}$ Rnase) incubated at $37^{\circ} \mathrm{C}$ with continuous shaking overnight. They were then placed into $3 \mathrm{ml}$ of ESP buffer containing proteinase $\mathrm{K}$ at $100 \mu \mathrm{g} / \mathrm{ml}$ and $1 \%$ SDS and incubated overnight at $55^{\circ} \mathrm{C}$ for $1 \mathrm{~h}$. The TE was replaced after $1 \mathrm{~h}$ with sterile, dilute TE and washed with gentle shaking for $1 \mathrm{~h}$. The TE was replaced with $2 \mathrm{ml}$ of fresh, dilute TE in a clean tube for storage at $4^{\circ} \mathrm{C}$ until restriction digestion. A plug slice 2 to $4 \mathrm{~mm}$ wide was placed in a tube with digestion mixture containing $25 \mathrm{ml}$ of restriction buffer (Gibco Bethesda Research Laboratories), $2 \mu \mathrm{l}(20 \mathrm{U}$ ) Sma1 (New England Biolab) and $200 \mu \mathrm{l}$ distilled water for $24 \mathrm{~h}$ at $25^{\circ} \mathrm{C}$. After digestion, plugs were loaded into a $1.2 \%$ agarose gel with $0.5 \mathrm{X}$ TBE, $89 \mathrm{mM}$ EDFTA and $0.05 \mu \mathrm{g} / \mathrm{ml}$ ethidium bromide using a CHEF-DR 11 apparatus (Bio-Rad Laboratories) at the following parameters: $14 \mathrm{C}, 200 \mathrm{~V}$, switching time $\mathrm{Si}=1 \mathrm{~s}, \mathrm{Sf}=20 \mathrm{c}$, for $21 \mathrm{~h}$.

\section{RESULTS}

The results of the antibiotic sensitivities determined by the agar disk diffusion and microdilution methods are shown in Tables 1 and 2. The only difference in susceptibility between the two 
Table I Susceptibility profile of GBS isolates*

\begin{tabular}{llcc}
\hline Antibiotic & Susceptible & Intermediate & Resistant \\
\hline Ampicillin & $43(62.7 \%)$ & $9(17.3 \%)$ & 0 \\
Azithromycin & $33(66.5 \%)$ & $3(5.8 \%)$ & $16(30.8 \%)$ \\
Cefamandole & $45(68.5 \%)$ & 0 & $7(13.5 \%)$ \\
Ceftriaxone & $26(50 \%)$ & 0 & $8(15.4 \%)$ \\
Ciprofloxacin & $30(68.2 \%)$ & $14(31.8 \%)$ & 0 \\
Clindamycin & $42(80.8 \%)$ & 0 & $10(19.2 \%)$ \\
Erythromycin & $34(65.4 \%)$ & $5(9.6 \%)$ & $13(25 \%)$ \\
Nitrofurantoin & $51(98.1 \%)$ & $1(1.9 \%)$ & 0 \\
Ofloxacin & $26(100 \%)$ & 0 & 0 \\
Penicillin G & $44(84.6 \%)$ & $8(15.4 \%)$ & 0 \\
Vancomycin & $52(100 \%)$ & 0 & 0
\end{tabular}

*Antibiotic sensitivity testing, both disk diffusion and microdilution methods, followed NCCLS guidelines. Antibiotic cut-off for susceptible and resistant not found in NCCLS guidelines for Streptococci; used Staphylococcus values as recommended by the NCCLS

Table 2 Microdilution susceptibility analyzes of GBS isolates determined to intermediate or resistant by disc diffusion method*

\begin{tabular}{lccc}
\hline & \multicolumn{3}{c}{ Microdilution* } \\
\cline { 2 - 4 } Disc diffusion & Susceptible & Intermediate & Resistant \\
\hline Penicillin G intermediate-susceptible & $2 / 10(20.0 \%)$ & $8 / 10(80.0 \%)$ & - \\
Ampicillin intermediate-susceptible & $10 / 19(52.6 \%)$ & $9 / 19(47.4 \%)$ & - \\
Clindamycin- resistant & - & - & $10 / 10(100 \%)$ \\
\hline
\end{tabular}

*According to $\mathrm{NCCLS}^{17}$

methods was found in response to ampicillin. Approximately one-half of the isolates classified as intermediate-susceptible to ampicillin by disk diffusion also were found to be susceptible by the microdilution method. However, eight of 10 strains classified as intermediate sensitive to penicillin $\mathrm{G}$ by agar disk diffusion method were found to maintain this decreased degree of sensitivity by the microdilution method. Of these, seven isolates displayed intermediate-sensitivity to ampicillin (Tables 3-5). All 10 isolates found to be resistant to clindamycin by disk diffusion were also resistant by the microdilution method, with a $\mathrm{MIC}_{90}$ $\geq 8 \mu \mathrm{g} / \mathrm{ml}$. Since no differences were found between the disk diffusion and microdilution methods, the former can be used when testing GBS isolates for antibiotic resistance. The only significant difference $(p \leq 0.019)$ in measurements of inhibition zones between the use of MuellerHinton agar, with and without 5\% sheep blood, was found with ciprofloxin.
Table 3 Comparison of the frequency of disc diffusion susceptible GBS isolates in Mueller-Hinton agar with and without $5 \%$ sheep blood*

\begin{tabular}{lccc}
\hline & $\begin{array}{c}\text { Mueller-Hinton } \\
\text { agar with 5\% } \\
\text { sheep blood }\end{array}$ & $\begin{array}{c}\text { Mueller-Hinton } \\
\text { agar without }\end{array}$ & \\
$5 \%$ sheep blood & $p+$ \\
\hline Ampicillin & $33 / 52(63.5 \%)$ & $32 / 52(61.5 \%)$ & NS \\
Azithromycin & $33 / 52(63.5 \%)$ & $33 / 52(63.5 \%)$ & NS \\
Cefamandole† & $45 / 52(86.5 \%)$ & $45 / 52(86.5 \%)$ & NS \\
Cefazolin† & $31 / 34(91.2 \%)$ & $32 / 34(94.1 \%)$ & NS \\
Ceftriaxone & $26 / 52(50.0 \%)$ & $30 / 52(57.7 \%)$ & NS \\
Ciprofloxacin† & $30 / 44(68.2 \%)$ & $19 / 44(43.2 \%)$ & 0.019 \\
Clindamycin & $42 / 52(80.8 \%)$ & $43 / 52(82.7 \%)$ & NS \\
Erythromycin & $34 / 52(65.4 \%)$ & $37 / 52(71.2 \%)$ & NS \\
Nitrofurantoin $\dagger$ & $51 / 52(98.1 \%)$ & $51 / 52(98.1 \%)$ & NS \\
Ofloxacin & $18 / 18(100 \%)$ & $16 / 18(88.9 \%)$ & NS \\
Penicillin G & $42 / 52(80.8 \%)$ & $43 / 52(82.7 \%)$ & NS \\
Vancomycin & $52 / 52(100 \%)$ & $52 / 52(100 \%)$ & NS \\
\hline
\end{tabular}

*According to $\mathrm{NCCLS}^{17}$

†Antibiotics not found in the NCCLS for Streptococci. Used Staphylococci values.

$+\mathrm{P}$ value based on McNemar test. NS = not significant 
Table 4 Resistance of GBS isolates to other antibiotics among the penicillin intermediate-susceptible strains

\begin{tabular}{lcccccc}
\hline ISS* & Azithromycin & Cefamandole & Cefazolin & Ceftriaxone & Clindamycin & Erythromycin \\
\hline Amp 9 & $4(44 \%)$ & $6(67 \%)$ & $8(89 \%)$ & $8(89 \%)$ & $7(78 \%)$ & $3(33 \%)$ \\
Pen 8 & $4(50 \%)$ & $6(75 \%)$ & $7(87 \%)$ & $7(87 \%)$ & $8(100 \%)$ & $2(25 \%)$ \\
\hline
\end{tabular}

Table 5 GBS isolates that exhibited resistance to more than $\mathrm{PNE}$ antibiotic

\begin{tabular}{ll}
\hline Antibiotics* & $n(\%)$ \\
\hline 1 & $9(33)$ \\
2 & $6(22)$ \\
3 & $5(19)$ \\
4 & $2(4)$ \\
5 & $4(8)$ \\
\hline
\end{tabular}

\section{DISCUSSION}

The use of intrapartum antibiotics to prevent perinatal vertical transmission of GBS and earlyonset neonatal sepsis has increased significantly since the Center for Disease Control (CDC) published guidelines in 1996 and subsequently released revised guidelines in $2002^{8,23}$. The current strategy for prevention estimates that approximately $24-27 \%$ of laboring women will receive intrapartum antibiotics $3,4,17,23,24$. An estimated 4 million births occur per year in the United States; therefore, approximately 1 million women will be at risk for GBS colonization and potentially receive intrapartum antibiotic prophylaxis. The antibiotic of choice is either penicillin $\mathrm{G}$ or ampicillin. The current CDC guidelines recommend that patients not allergic to penicillin receive penicillin or ampicillin. Individuals with a minor allergy to penicillin should receive cefazolin and individuals with a major allergy (rash or a history of difficult breathing) should receive clindamycin or vancomycin if the isolate is known to be resistant to clindamycin. However, vancomycin has not been shown to cross the placenta and achieve suitable concentrations in amniotic fluid as well as the fetal blood. This tremendous use of $\beta$-lactam antibiotics coupled with the exposure to $\beta$-lactams for other reasons can potentially induce the emergence of resistant strains among the resident vaginal microflora. Emergence of resistance has been documented, especially to ampicillin, e.g. Escherichia coli $^{16-18}$. However as documented in several studies, GBS is universally sensitive to the penicillins; therefore, it should be the primary antibiotic for intrapartum prophylaxis ${ }^{3,4}$.

In this study, as in other studies, strains of penicillin-resistant GBS were not found. However, the isolates demonstrated an intermediate sensitivity to penicillin $\mathrm{G}$ in $17 \%$ of cases and to ampicillin in $15 \%$ of cases using both the disk diffusion and micro-tube dilution methods for determining antibiotic sensitivity. In fact, the disk diffusion method revealed that $19 \%$ of the isolates had an intermediate sensitivity to penicillin while $36 \%$ had an intermediate sensitivity to ampicillin. Rouse et al. reported that $10 \%$ and $8 \%$ of the isolates they tested had intermediate susceptibility to penicillin and ampicillin respectively ${ }^{17}$. Betriu et al. reported that $2 \%$ of GBS isolates had an intermediate susceptibility to penicillin ${ }^{16}$. Liu et al. found that $15 \%$ of the GBS isolates tested had an intermediate susceptiblity to penicillin ${ }^{18}$. The finding of strains with an intermediate susceptibility to penicillin $G$ and ampicillin does not mean that these antibiotics should not be used. However, this data does indicate that GBS isolates should be monitored annually to determine if a decrease in susceptibility emerges, thus leading to resistance. The continued use of large amounts of $\beta$-lactams will lead to resistance as it has in Streptococcus pneumoniae.

Furthermore, it has been suggested that the significant decrease in the incidence of GBS neonatal sepsis may be accompanied by a rise in the incidence of Gram-negative neonatal sepsis. Levine et al. examined the incidence of GBS and Gram-negative neonatal sepsis prior and subsequent to the CDC recommendations. They found that while the incidence of GBS sepsis declined significantly, the incidence of Gram-negative neonatal sepsis increased 4.5 fold in the same period. Another important observation was that in all cases of neonatal death caused by sepsis, the mothers received intrapartum prophylaxis with ampicillin and the pathogens recovered from the 
neonates were resistant to ampicillin ${ }^{3}$. These findings were similar to other reported cases of adverse perinatal outcomes with resistant Enterobacteriaceae after antibiotic usage for premature rupture of membranes and carriage of $\mathrm{GBS}^{25}$.

Conversely, the data reported by Meyn and Hillier did not suggest a trend toward reduced susceptibility for vaginal GBS and E. coli isolates to ampicillin. They did not find intermediatesusceptible GBS or any differences in the percentage of ampicillin-resistant $E$. coli during three years ${ }^{5}$. Despite these results, some authors have suggested penicillin $G$ is a better choice than ampicillin for GBS intrapartum prophylaxis. Penicillin $G$ has a narrow spectrum of activity and may be less likely to select resistant microorganisms than ampicillin ${ }^{3,26}$.

In the present study GBS isolates were resistant to: clindamycin (19\%), erythromycin (25\%) and cefazolin (15\%). These results are not very different from previous reports regarding clindamycin and erythromycin resistance ${ }^{16,17}$. However, the rate of resistance reported in this study was higher than that reported in other studies. The parameters used in other studies are not known because the NCCLS has not published guidelines regarding sensitivity and resistance for cefazolin for GBS. The values used in this study were those used for Staphylococcus species because of the similarity to GBS and recommended by the NCCLS. In addition, to ensure that the isolates were not misidentified as Enterococcus species, the latex agglutination antibody-antigen test specific for GBS was used to confirm that the isolate was GBS.

Representatives of second- and thirdgeneration cephalosporins were also used, e.g. cefamandole and ceftriaxone. These antibiotics also demonstrated a high level of resistance (13\% and $30 \%$ ) and do not appear to be good alternatives to penicillin. The ability of cephalosporins to prevent vertical transmission of neonatal GBS infection has not been studied ${ }^{4}$.

Other antibiotics tested as possible alternatives to penicillin showed advantages regarding GBS susceptibility. In agreement with other published studies all isolates tested in this study were susceptible to vancomycin ${ }^{4,16}$. This antibiotic is currently the alternative to penicillin in the patient with a known allergy to penicillin and is also colonized with a strain of GBS that is resistant to clindamycin. However the potential toxicity and concerns about selection of resistance, especially to Gram-positive cocci, do not make widespread use of vancomycin a good alternative at this time. Preliminary data suggest that vancomycin does cross the placenta and achieves adequate blood levels in the fetus (personal communication).

The susceptibility of GBS was tested against nitrofurantoin because it is a good option for the treatment of both asymptomatic and symptomatic bacteriuria. Reports regarding the susceptibility pattern of a large number of GBS isolates to nitrofurantoin have not been published. No resistance to nitrofurantoin was found in the GBS isolates tested in the current study. Unfortunately, nitrofurantoin cannot be used as an alternative to penicillin for intrapartum prophylaxis because effective concentrations are not likely to be achieved in the maternal blood stream or to cross the placenta to achieve adequate levels in the fetal compartment. However, nitrofurantoin is suitable to treat bacteriuria in the pregnant patient because of the high levels achieved in the urine. It also has a good spectrum of activity against Gram-negative bacteria including E. coli. Nitrofurantoin is unlikely to have a significant impact on the endogenous resident microflora and therefore, it is unlikely that it will select for resistant strains.

In summary, it is not known whether the antibiotic resistance demonstrated in vitro has the same clinical significance in vivo. Future clinical trials are needed to identify safe and effective alternatives to penicillin for intrapartum prophylaxis to prevent perinatal vertical transmission and infection with GBS. There are probably important regional variations in GBS antibiotic susceptibilities, especially for second antibiotics. Therefore, in the penicillin-allergic patient, the laboratory should be requested to perform antibiotic sensitivities on the GBS isolate. This will enable the physician to choose an appropriate alternative to penicillin. 


\section{REFERENCES}

1. Schuchat A. Epidemiology of group B streptococcal disease in the United States: shifting paradigms. Clin Microbiol Rev 1998;11:497-513

2. Harrison LH, Ali D, Dwyer DM, et al. Relapsing invasive group B streptococcal infection in adults. Ann Internal Med 1995;123:421-7

3. Levine EM, Ghai V, Barton JJ, Strom CM. Intrapartum antibiotic prophylaxis increases the incidence of gram-negative neonatal sepsis. Infect Dis Obstet Gynecol 1999;7:210-3

4. Pearlman MD, Pierson CL, Faix RG. Frequent resistance of clinical group B streptococci isolates to clindamycin and erythromycin. Obstet Gynecol 1998;92:258-61

5. Meyn LA, Hillier SL. Ampicillin susceptibilities of vaginal and placental isolates of group B streptococcus and Escherichia coli obtained between 1992 and 1994. Antimicrob Agents Chemother 1997;41: 1173-4

6. Lin L, Song J, Kimber N, et al. The role of bacterial vaginosis in infection after major gynecologic surgery. Infect Dis Obstet Gynecol 1999;7:169-74

7. Scharg SJ, Zywicki S, Farley MM, et al. Group B streptococcal disease in the era of intrapartum antibiotic prophylaxis. N Engl J Med 2000;342:15-20

8. Anonymous. Early-Onset Group B Streptococcal Disease - United States, 1998-1999. MMWR Morb Mortal Wkly Rep 2000;49:793-6

9. Tyrrell GJ, Senzilet LD, Spika JS, et al. Invasive disease due to group B streptococcal infection in adults: results from a Canadian, population-based, active laboratory surveillance study - 1996. J Infect Dis 2000;182:168-73

10. Schwartz B, Schuchat A, Oxtoby MJ, et al. Invasive group $\mathrm{B}$ streptococcal disease in adults. J Am Med Assoc 1991;266:1112-4

11. Farley MM, Harvey RC, Stull T, et al. A population-based assessment of invasive disease due to group B Streptococcus in non-pregnant adults. N Engl J Med 1993;328:1807-11

12. Blumberg HM, Stephens DS, Modanksy M, et al. Invasive group B streptococcal disease: the emergence of serotype V.J Infect Dis 1996;173: 365-73

13. Wessels MR, Kasper DL. The changing spectrum of group B streptococcal disease (editorial). NEngl J Med 1993;328:1843-4

14. Anonymous. Prevention of perinatal group B streptococcal disease: a public health perspective.
Centers for Disease Control and Prevention. MMWR Morb Mortal Wkly Rep 1996;45:1-24

15. American College of Obstetricians and Gynecologists. Prevention of early-onset group B streptococcal disease in newborns. American College of Obstetricians and Gynecologists, 1996

16. Betriu C, Gomez M, Sanchez A, et al. Antibiotic resistance and penicillin tolerance in clinical isolates of group B streptococci. Antimicrob Agents Chemother 1994;38:2183-6

17. Rouse DJ, Andrews WW, Lin FC, et al. Antibiotic susceptibility profile of group B Streptococcus acquired vertically. Obstet Gynecol 1998;92:931-4

18. Liu JW, Wu JJ, Ko WC, Chuang YC. Clinical characteristics and antimicrobial susceptibility of invasive group B streptococcal infection in nonpregnant adults in Taiwan. J Formos Med Assoc 1997;96:628-33

19. Fernandez M, Hickman ME, Baker CJ. Antimicrobial susceptibilities of group B streptococci isolated between 1992 and 1996 from patients with bacteremia or meningitis. Antimicrob Agents Chemother 1998;42:1517-9

20. National Committee for Clinical Laboratory Standards. Performance Standards for Antimicrobial Disk Susceptibility Tests, sixth edn; approved standard. NCCLS document M10A6, vol. 17, no. 1, Wayne, PA: National Committee for Clinical Laboratory Standards, 1997

21. National Committee for Clinical Laboratory Standards. Performance Standards for Antimicrobial Susceptibility Testing; Ninth Informational Supplement. NCCLS Document M100-S9, vol. 19, no. 1, Wayne, PA: National Committee for Clinical Laboratory Standards, 1999

22. Matushek MG, Bonten MJ, Hayden MK. Rapid preparation of bacterial DNA for pulsed-field gel electrophoresis. J Clin Microbiol 1996;34:2598-600

23. Prevention of Perinatal Group B Streptococcal Disease. Revised Guidelines from the CDC. MMWR Morb Mortal Wkly Rep 2002;51(RR11): $1-22$

24. Baker CJ, Edwards MS. Group B streptococcal infections. In Remington J, Klein LO, eds. Infectious diseases of the fetus and newborn infant. Philadelphia: W.B. Saunders, 2001;742-811

25. McDuffie RS, McGregor JA, Gibbs RS. Adverse perinatal outcome and resistant 
Enterobacteriaceae after antibiotic usage for premature rupture of membrane and group $\mathrm{B}$ streptococcus carriage. Obstet Gynecol 1993;82: 487-9

ReCEIVED 11/12/01; ACCEPTED 14/04/04
26. Amstey MS, Gibbs RS. Is penicillin G a better choice than ampicillin for prophylaxis of neonatal group B streptococcal infections? Obstet Gynecol 1994;84:1058-9 


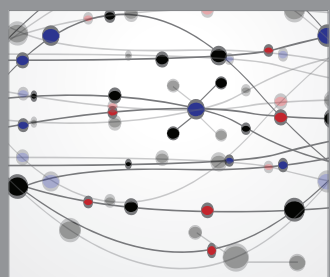

The Scientific World Journal
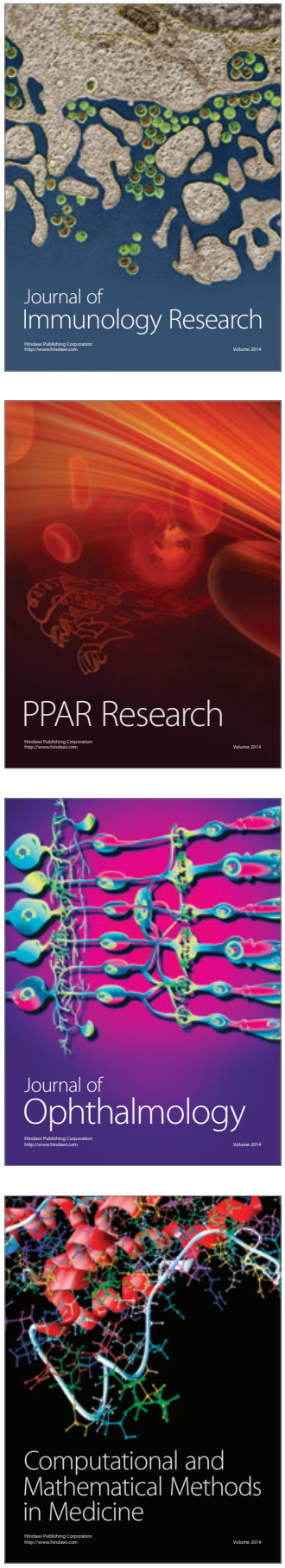

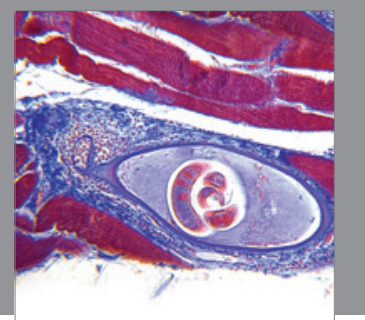

Gastroenterology

Research and Practice
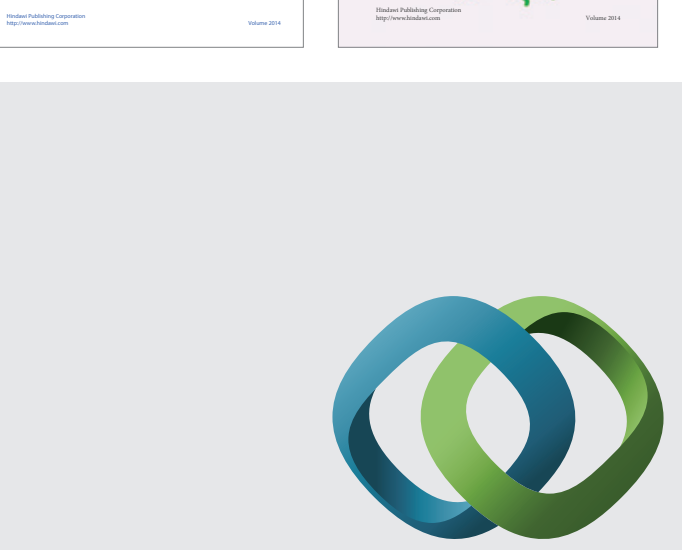

\section{Hindawi}

Submit your manuscripts at

http://www.hindawi.com
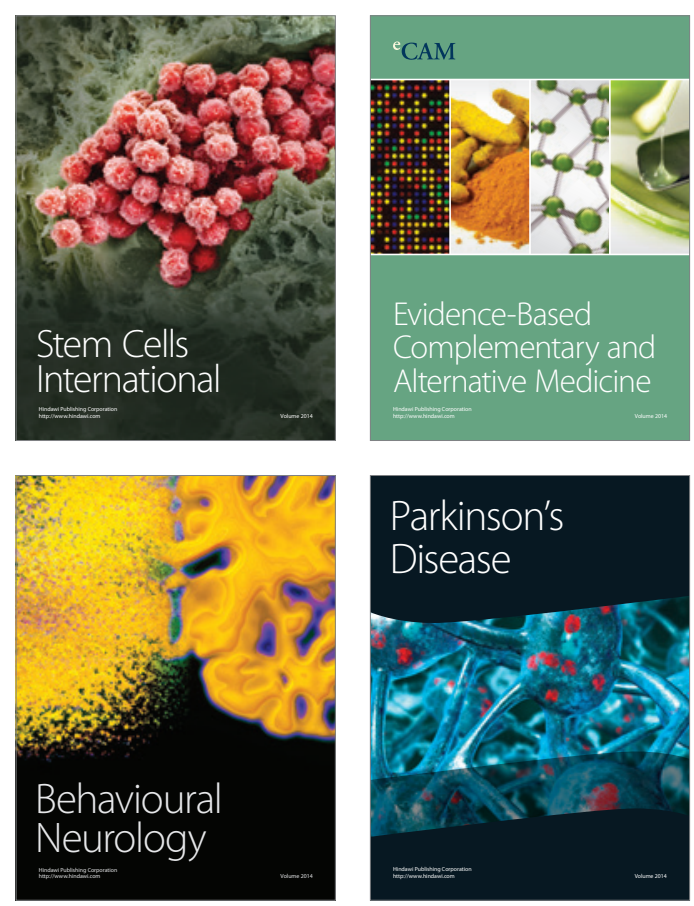

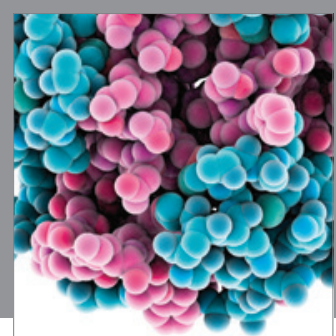

Journal of
Diabetes Research

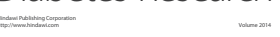

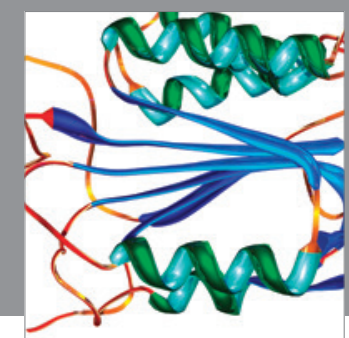

Disease Markers
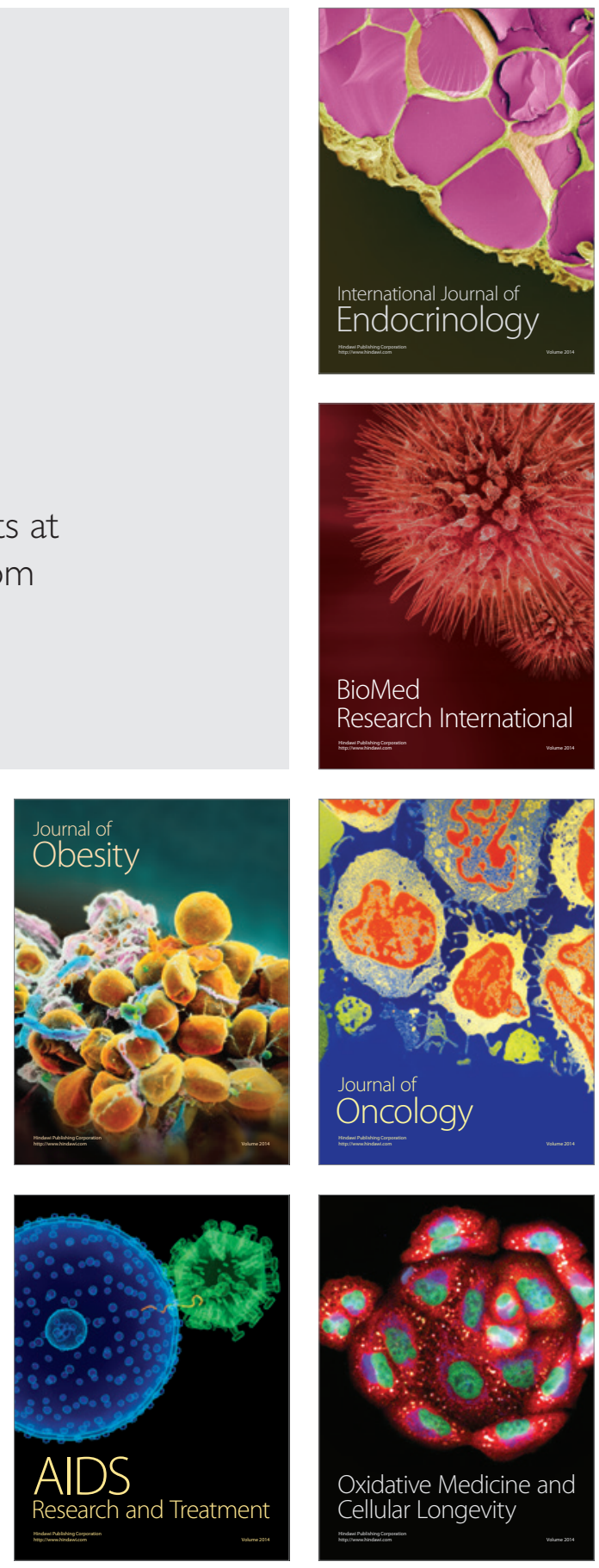